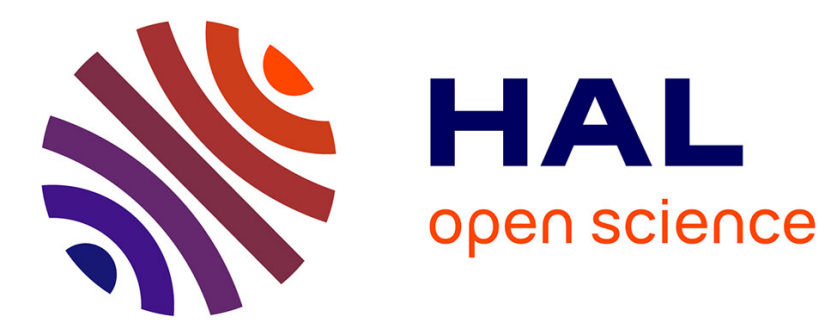

\title{
Jean-Jacques Rousseau et le symbolisme du pouvoir Patrice Canivez
}

\section{To cite this version:}

Patrice Canivez. Jean-Jacques Rousseau et le symbolisme du pouvoir. Marie-Madeleine Castellani et Fiona McIntosh-Varjabédian. Représenter le pouvoir. Images du pouvoir dans la littérature et les arts, 28, P.I.E. Peter Lang, pp.409-421, 2014, Comparatisme et société, 978-2-87574-179-0. hal-01091389

\section{HAL Id: hal-01091389 \\ https://hal.science/hal-01091389}

Submitted on 5 Dec 2014

HAL is a multi-disciplinary open access archive for the deposit and dissemination of scientific research documents, whether they are published or not. The documents may come from teaching and research institutions in France or abroad, or from public or private research centers.
L'archive ouverte pluridisciplinaire HAL, est destinée au dépôt et à la diffusion de documents scientifiques de niveau recherche, publiés ou non, émanant des établissements d'enseignement et de recherche français ou étrangers, des laboratoires publics ou privés. 


\title{
Jean-Jacques Rousseau et le symbolisme du pouvoir
}

\author{
Patrice Canivez
}

Chapitre de l'ouvrage Représenter le pouvoir. Images du pouvoir dans la littérature et les arts, MarieMadeleine Castellani et Fiona McIntosh-Varjabédian (Dir.), Bruxelles, P.I.E. Peter Lang, 2014, pp. 409-421 (pagination originale insérée en gras dans le texte).

Pour traiter la question du symbolisme du pouvoir chez Rousseau, il faut partir de sa théorie du signe et de l'usage qu'il fait lui-même des symboles. Il faut aussi faire la distinction entre le pouvoir souverain et le pouvoir du prince. Le symbole, chez Rousseau, est un acte de communication qui donnez à penser en donnant à voir. Il agit sur l'interlocuteur de deux façons différentes qui peuvent être complémentaires. D'une part, il donne à la communication un maximum de force en condensant la signification dans un geste, une chose ou une image. D'autre part, il crée chez l'interlocuteur la disposition d'esprit adéquate pour comprendre et accepter le discours qui lui est adressé. Le point commun à ces deux modalités du symbole comme acte, c'est qu'elles relèvent de la persuasion plutôt que de la conviction, de la force de l'expression plutôt que de l'argumentation.

Quant à la distinction entre le souverain et le prince, elle correspond à la différence entre le législatif et l'exécutif. Dans un État fondé sur le contrat social, autrement dit, dans un Etat légitime, le peuple est le seul souverain. La souveraineté populaire consiste dans le pouvoir de faire la loi, qui est l'expression de la volonté générale. En revanche, l'exécution des lois est confiée à un gouvernement qui peut être de forme démocratique, aristocratique, monarchique, ou d'une forme mixte combinant des éléments de ces divers régimes. Mais quelle qu'en soit la forme, Rousseau désigne le gouvernement par l'expression : le prince ${ }^{1}$. Il y a donc deux pouvoirs hiérarchisés : le pouvoir souverain et le pouvoir du prince. Si le prince est un monarque, ce monarque doit reconnaître le peuple comme seul souverain légitime. S'il se déclare souverain lui-même, sa souveraineté est par là-même usurpée. Ce pouvoir peut (410) s'imposer par la contrainte, mais il n'est pas légitimé par le contrat social.

Dans la présentation qui suit, je commencerai par proposer des remarques sur les deux modes d'action du symbole, en partant de l'Essai sur l'origine des langues et de la Profession de foi du vicaire savoyard. Puis, j'envisagerai la question du symbolisme du pouvoir en m'attachant successivement au pouvoir souverain et au pouvoir du prince. Au cours de cet exposé, je tiendrai également compte d'une troisième distinction : la distinction entre la place du symbolisme dans l'action publique et l'usage de l'expression symbolique par Rousseau en tant qu'écrivain politique. Sur ce point, il y a une importante remarque à faire. Il me semble en effet que la plupart des symboles qu'utilise Rousseau, à la fois comme éléments de sa théorie de l'État et comme forme d'expression, ont la particularité d'agir en procédant à des inversions de sens. Le symbolisme que Rousseau met en place agit de façon paradoxale. Il prend l'opinion commune à contrepied. L'esprit de l'interlocuteur ou du lecteur n'est pas

\footnotetext{
${ }^{1}$ Cf. Du contrat social, Livre III, chapitre I, Euvres complètes, T. III, Paris, Gallimard, Bibliothèque de la Pléiade, 1964, p. 396.
} 
seulement comme arrêté, il est contraint de repartir dans l'autre sens. La fonction du symbole est ici de provoquer une conversion de l'esprit. Je terminerai sur ce point en mettant en parallèle, sur l'exemple de l'approche rousseauiste de la monarchie, un usage normatif et un usage critique du symbole.

\section{Le symbole et l'expression du sens}

\section{I.1. La communication des significations}

Ce que nous appelons «symbole» a pour modèle, chez Rousseau, les «signes » qui relèvent de la «langue du geste». Un passage de l'Essai sur l'origine des langues nous permettra de voir ce dont il s'agit.

Quoique la langue du geste et celle de la voix soient également naturelles, toutefois la première est plus facile et dépend moins des conventions : car plus d'objets frappent nos yeux que nos oreilles et les figures ont plus de variété que les sons; elles sont aussi plus expressives et disent plus en moins de temps [...]. Ce que les anciens disaient le plus vivement, ils ne l'exprimaient pas par des mots mais par des signes ; ils ne le disaient pas, ils le montraient [...]. Ainsi l'on parle aux yeux bien mieux qu'aux oreilles (...). On voit même que les discours les plus éloquents sont ceux où l'on enchâsse le plus d'images, et les sons n'ont jamais plus d'énergie que quand ils font l'effet des couleurs. ${ }^{1}$

(411) Le propre du geste, c'est qu'il concentre la signification dans ce qu'il donne à voir, au lieu de déployer cette signification dans un discours articulé. Du fait que le sens est condensé dans l'unité d'une image, dans une concentration du temps et de l'espace, il acquiert une force et une énergie que ne possède pas le discours développé. Il y a donc une relation inversement proportionnelle entre la force de l'expression et sa rigueur logique. La signification exhibée dans un geste est plus forte, mais elle n'est pas explicitée. En revanche, ce que le discours gagne en articulation logique, il le perd en force d'expression. L'idéal de la rhétorique rousseauiste, c'est donc un discours qui aurait à la fois la rigueur du raisonnement et la force expressive du geste. C'est un discours qui aurait, à la fois, la capacité de convaincre par des raisons et celle de persuader par des images qui agissent comme des gestes. On retrouve ici l'idéal rousseauiste de la communication immédiate, de la transparence entre le locuteur et l'interlocuteur, entre celui qui exhibe le geste et celui qui l'observe. Or, la communication est d'autant plus immédiate qu'elle est plus ramassée ; et plus elle est ramassée, plus elle agit.

De cette théorie du signe qui montre plus qu'il ne démontre, Rousseau donne des exemples tirés de la « communication politique »:

Ouvrez l'histoire ancienne vous la trouverez pleine de ces manières d'argumenter aux yeux, et jamais elles ne manquent de produire un effet plus assuré que tous les discours qu'on aurait pu mettre à la place : l'objet offert avant de parler ébranle l'imagination, excite la curiosité, tient l'esprit en suspens et dans l'attente de ce qu'on va dire [...] le langage le plus énergique est celui où le signe a tout dit avant qu'on parle [...]. Darius engagé dans la Scythie avec son armée reçoit de la part du Roi des Scythes une grenouille, un oiseau, une souris et cinq flèches : le héraut remet son présent en silence et part. Cette terrible harangue fut entendue, et Darius n'eut plus grande hâte

\footnotetext{
${ }^{1}$ Essai sur l'origine des langues, chapitre I, «Des divers moyens de communiquer nos pensées », Euvres complètes, T. V, Paris, Gallimard, Bibliothèque de la Pléiade, 1995, pp. 376-377.
} 
que de regagner son pays comme il put. Substituez une lettre à ces signes, plus elle sera menaçante, moins elle effrayera : ce ne sera plus qu'une gasconnade dont Darius n'aurait fait que rire. ${ }^{1}$

\section{I.2. La communication du sentiment et la disposition d'esprit}

Il y a cependant un autre fonctionnement du symbole chez Rousseau, c'est le symbole qui crée, chez l'interlocuteur ou le spectateur, une disposition subjective qui le prépare à accepter une idée, une thèse, en s'assimilant l'état d'esprit dans lequel cette idée ou cette thèse ont été formulées. De ce travail sur la « réception » du discours, on trouve un exemple au début de la Profession de foi du vicaire savoyard. Certes, nous ne sommes pas avec la Profession de foi dans le domaine de la politique (412), mais celle-ci nous met en présence d'une sorte de paradigme. On se souvient que la Profession de foi met en scène un dialogue entre le vicaire et son jeune interlocuteur. Le but du vicaire est d'exposer ses raisons de croire en Dieu. Mais les raisons qu'il invoque ne sont pas tant un moyen de convaincre. Elles sont plutôt l'expression d'un sentiment: celui d'une foi authentique. Il s'agit moins de démontrer quelque chose que de communiquer un sentiment. Or, communiquer un sentiment n'est pas transmettre un message. C'est l'exprimer en des termes propres à éveiller un sentiment analogue dans l'esprit de l'interlocuteur. C'est le formuler de telle manière que l'interlocuteur soit amené à faire retour sur lui-même, à examiner si le discours qui lui est adressé trouve un écho dans ses propres convictions intimes. Dit autrement, il s'agit de communiquer un état d'esprit en provoquant, chez le destinataire, l'éveil de cet état d'esprit. Si l'on y parvient, les arguments acquièrent une force de conviction qu'ils n'ont pas lorsqu'ils sont exposés abstraitement, de façon purement rationnelle.

Comment s'y prend-on? D'abord, il y a le style de l'expression. Il faut écarter les artifices rhétoriques et les raisonnements subtils - en l'occurrence, les abstractions de la métaphysique - pour faire du discours l'expression aussi naturelle que possible de la conviction. Mais il y a aussi le symbolisme du lieu et du moment. Pour communiquer un certain état d'esprit, il ne faut pas parler à n'importe quel moment, il ne faut pas parler dans n'importe quel endroit. Dans le cas de la Profession de foi, ce que le vicaire savoyard veut transmettre à son jeune auditeur, c'est la religion naturelle. C'est la foi que l'on acquiert, non pas en lisant les textes sacrés, mais en consultant son propre cœur. Pour éveiller le sentiment naturel, il faut alors écarter les livres et les dogmes, et choisir pour cadre de l'entretien la nature elle-même. Il faut choisir le lieu : la campagne. Il faut aussi choisir le moment : le lever du jour. Le moment où la nature s'éveille est le moment propice pour éveiller le sentiment naturel, c'est-à-dire, pour retrouver la vérité qui se délivre dans la conviction authentique.

Voici donc le prologue de la Profession de foi du vicaire savoyard:

On était en été ; nous nous levâmes à la pointe du jour. Il me mena hors de la ville, sur une haute colline au dessous de laquelle passait le Pô, dont on voyait le cours à travers les fertiles rives qu'il baigne. Dans l'éloignement, l'immense chaîne des Alpes couronnait le paysage. Les rayons du soleil levant rasaient déjà les plaines, et projetant sur les champs par longues ombres les arbres, les coteaux, les maisons, enrichissaient de mille accidents de lumière le plus beau tableau dont l'œil humain puisse être frappé. On eût dit que la nature étalait à nos yeux toute sa magnificence pour en

\footnotetext{
${ }^{1}$ Ibid.
} 
offrir le texte à nos entretiens. Ce fut là, qu'après avoir quelque temps contemplé ces objets en silence, l'homme de paix me parla ainsi :...' ${ }^{1}$.

(413) Le spectacle de la nature doit créer, dans l'âme de l'interlocuteur du vicaire, la disposition d'esprit appropriée pour recevoir et comprendre son discours, pour laisser s'éveiller en lui la conviction que ce discours veut communiquer. Car le discours du vicaire est une réfutation de l'athéisme, et plus particulièrement, du matérialisme. La nature, ce n'est pas seulement la matière; et l'ordre de la nature, ce n'est pas un simple mécanisme. Or, ce que le vicaire va exposer est déjà exprimé par la nature elle-même. C'est pourquoi Rousseau dit que la nature est un texte. Le dialogue porte, d'une certaine manière, sur l'interprétation de ce texte. Par conséquent, la nature symbolise. Mais ce qu'elle symbolise, c'est elle-même. La beauté de la nature est le symbole de son irréductibilité à la matière et à la mécanique des forces.

\section{Le symbolisme et l'exercice du pouvoir.}

Il s'agit maintenant d'appliquer au symbolisme du pouvoir les trois remarques que nous avons faites au sujet du symbole chez Rousseau. Tout d'abord, le symbole agit sur l'esprit de l'interlocuteur par la force même de l'acte de communication. Il signifie le plus en disant le moins. Il a d'autant plus d'effet qu'il est signifiant. Il s'oppose en ce sens à la démonstration qui perd en force ce qu'elle gagne en clarté. Ensuite, le symbole crée chez l'interlocuteur la disposition d'esprit propre à recevoir et à accepter certaines significations. Enfin, l'usage que Rousseau lui-même fait des symboles consiste à provoquer des inversions de sens, une sorte de conversion de la manière de penser.

\section{II.1. Le peuple souverain}

Qu'en est-il dans les États républicains, c'est-à-dire, dans les États où le peuple est le vrai souverain ? Il y ici a trois éléments à prendre en compte : d'une part, l'autoreprésentation du peuple souverain; d'autre part, la fonction théologico-politique du symbolisme religieux; enfin, la fonction identitaire du symbolisme culturel.

\section{II.1.1. L'autoreprésentation du peuple}

Dans un État fondé sur le contrat social, il ne peut pas y avoir à proprement parler de représentation du pouvoir souverain. Et cela, qu'on entende la représentation au sens de délégation ou au sens de figuration. D'une part, le pouvoir souverain - celui de faire la loi ne se représente pas dans le sens où il ne peut pas se déléguer (414). Le peuple doit exercer lui-même l'autorité législative. Il ne doit pas y avoir de députés et s'il y en a, ces députés doivent être liés par un mandat impératif ${ }^{2}$. D'autre part, il ne peut pas y avoir de représentation au sens de figuration du pouvoir. Il ne peut pas y avoir de représentation du pouvoir à l'usage du peuple, puisque le peuple est cela-même qu'il faudrait représenter. Figuration du pouvoir et figuration du peuple sont une seule et même chose, puisque peuple et pouvoir ne sont pas séparés. Il ne peut donc y avoir qu'une représentation du peuple par lui-

\footnotetext{
${ }^{1}$ Emile, Livre IV, Euvres complètes, T. IV, Paris, Gallimard, Bibliothèque de la Pléiade, 1969, p. 565.

${ }^{2}$ Cf. Du contrat social, Livre III, chapitre XV, « Des députés ou représentants », o.c., pp. 429-430.
} 
même et à ses propres yeux. Cette autoreprésentation du peuple souverain a notamment lieu dans les fêtes civiques et populaires. L'évocation de la fête à Saint-Gervais, à la fin de la Lettre à d'Alembert, en est une illustration ${ }^{1}$. En analysant ce passage dans La transparence et l'obstacle, Jean Starobinski a montré que la fête est une manifestation dont la dynamique correspond à la structure du contrat social : chacun est reconnu par tous les autres, chacun se sait reconnu par tous et se reconnaît dans le peuple ${ }^{2}$. Cette fonction symbolique de la fête nationale et civique, comme on le sait, sera reprise par les révolutionnaires français.

Cela dit, il y aura bien un usage politique du symbole relativement au peuple. Mais ce qui est en question, ce n'est pas tant l'exercice de la souveraineté que l'institution du peuple en tant que tel et son identité historique. D'un côté, il y aura un symbolisme théologico-politique dont la fonction est de sacraliser le contrat social et les lois fondamentales qui « instituent » le peuple. De l'autre, il y aura un symbolisme culturel dont le rôle est de cristalliser un sentiment d'appartenance autour d'une représentation de l'identité nationale. Dans les deux cas, il s'agit de pallier les limites d'une adhésion abstraite, purement rationnelle aux principes constitutifs de la République. D'une part, il s'agit de fonder le respect du contrat sur le sens du sacré ; d'autre part, il s'agit de fonder le patriotisme sur le sentiment de l'identité et de la continuité historique de la nation. Il s'agit d'amener les citoyens à faire "par goût et par passion, ce qu'on ne fait jamais assez bien quand on ne le fait que par devoir ou par intérêt $»^{3}$.

\section{II.1.2. La fonction théologico-politique du symbolisme religieux (415)}

Le peuple, chez Rousseau, ne fait que donner son consentement aux lois. Dans le cas des lois fondamentales, c'est-à-dire, de la constitution qui doit doter l'État d'un régime politique, celui qui propose est le «Législateur » dont Rousseau imagine la figure à partir des modèles antiques : Lycurgue à Sparte, Moïse pour les Hébreux, Numa à Rome ${ }^{4}$. Quand il s'agit de modifier les lois existantes, c'est le gouvernement qui propose au peuple de nouvelles lois ${ }^{5}$. Or, l'adoption des lois fondamentales de l'État pose un problème. Le Législateur ne peut pas faire accepter par des arguments ce qu'il propose, car les facultés morales et intellectuelles, le civisme et le sens politique, ne se développent que dans le cadre de l'État républicain. On ne peut pas les supposer acquises au moment où il s'agit de fonder cet État. Par conséquent, le Législateur ne pourra pas convaincre par des arguments ; il ne pourra pas non plus, cela va de soi, user de contrainte. Il ne pourra donc que persuader par des " signes » qu'il est revêtu d'une autorité spéciale. Ces signes sont des signes religieux. C'est en s'attribuant une mission divine que les grands législateurs, tel Moïse, persuadent le peuple d'adopter certaines lois fondamentales.

En d'autres termes, le symbolisme religieux est nécessaire pour sacraliser les lois. Il faut donner aux lois un caractère sacré afin qu'elles soient respectées tant que les citoyens ne sauront pas leur donner une adhésion raisonnée. Ce ne sont d'ailleurs pas seulement les lois qui doivent être considérées comme sacrées, c'est d'abord le contrat social lui-même. C'est pourquoi, dans la formulation du contrat retenue pour la Corse, formules républicaines et formules religieuses sont étroitement - et pour nous, curieusement - mêlées :

\footnotetext{
${ }^{1}$ Cf. Lettre à d'Alembert, Euvres complètes, T. V, o.c., p. 123-124, note de Rousseau.

${ }^{2}$ Jean Starobinski, La transparence et l'obstacle, Paris, Gallimard, 1971, p. 116-121.

${ }^{3}$ Considérations sur le gouvernement de Pologne, Euvres complètes, T. III, o.c., p. 961.

${ }^{4} \mathrm{Cf}$. Du contrat social, Livre II, ch. VII, « Du Législateur », et Considérations sur le gouvernement de Pologne, o.c., p. 956-968.

${ }^{5}$ Du contrat social, Livre IV, ch. I, « Que la volonté générale est indestructible », o.c., p. 439.
} 
Formule du serment prononcé sous le ciel et la main sur la Bible : Au nom de Dieu tout puissant et sur les saints Évangiles par un serment sacré et irrévocable je m'unis de corps, de biens, de volonté et de toute ma puissance à la nation corse [...]. Vive à jamais la liberté, la justice et la République des Corses. Amen ${ }^{1}$.

Il reste que le symbolisme religieux est attaché à une religion historique : judaïsme, christianisme, etc. Or, les religions posent un problème. Plus précisément, le christianisme en tant que religion instituée pose un problème. D'un côté, c'est un facteur de cohésion sociale et d'unité politique (416). C'est pourquoi le vicaire savoyard insiste sur le fait qu'il faut vivre dans la religion de son pays. Mais d'un autre côté, le christianisme est fondé sur un universalisme qui ne lui permet pas de distinguer un peuple parmi les autres, qui donc en fait un principe faible d'unité et de cohésion. D'autant que le christianisme repose sur l'opposition entre le temporel et le spirituel. La vraie fidélité du chrétien va au monde spirituel, non à la patrie temporelle. Enfin, la déviation qui fait de l'Église universelle un empire temporel, avec le pape comme souverain, introduit un irréconciliable conflit d'allégeances dans les États où cette Église est influente. Dès lors, le christianisme ne peut pas jouer le rôle de religion civile, du moins pas sur le modèle antique. Il faut donc envisager un nouveau modèle de religion civile, un modèle dans lequel la religion se résume à un petit nombre de dogmes rationnels. Il s'agit en fait d'un équivalent, dans le domaine politique, de la religion naturelle du vicaire savoyard. Puisqu'il ne s'agit pas de faire le choix de telle ou telle religion historique pour faire l'unité de l'Etat, la cohésion du corps politique doit reposer sur un tout autre principe, celui de la tolérance. Dans cette perspective, les dogmes que tous les citoyens seront censés admettre sont des dogmes très généraux, admissibles par les croyants de toute obédience : «l'existence de la divinité [...], la vie à venir, le bonheur des justes, le châtiment des méchants, la sainteté du contrat social et des lois $»^{2}$. Dans cette religion rationnelle, la sacralisation du contrat et des lois prendra une forme elle-même plus épurée. Le rôle du symbolisme tendra à décroître, ou bien la fonction politique du symbolisme sera moins importante.

\section{II.1.3. La fonction identitaire du symbolisme culturel}

Mais si la fonction théologico-politique du symbolisme du pouvoir tend à s'estomper, c'est au profit de la fonction identitaire du symbolisme culturel. C'est du moins le cas dans les Considérations sur le gouvernement de Pologne. Dans ce texte rédigé à la veille du premier partage de la Pologne ${ }^{3}$, Rousseau s'attache aux moyens d'assurer la survie de la nation polonaise. Les recommandations qu'il fait pour une réforme de l'État sont donc fortement liées à la question de sa continuité historique. Mais au-delà de ce cas particulier, Rousseau définit un modèle de construction de l'identité nationale qui deviendra prédominant au siècle suivant. Cette fois-ci, il s'agit moins de la sacralisation des lois que de la représentation de la nation en tant que «personnalité » historique. Ici, il ne s'agit pas de représenter le peuple en tant que souverain (417). Il ne s'agit pas non plus de fonder le respect des lois sur le sens du sacré. Il s'agit de cristalliser un sentiment d'appartenance collective autour d'une

\footnotetext{
${ }^{1}$ Constitution pour la Corse, Fragments séparés, Euvres complètes, T. III, o.c., p. 943.

${ }^{2}$ Cf. Du contrat social, Livre IV, chapitre VIII, « De la religion civile », o.c., p. 468.

${ }^{3}$ Les Considérations sur le gouvernement de Pologne ont été rédigées en 1770-1771, le premier partage de la Pologne entre 1'Autriche, la Prusse et la Russie intervient en 1772.
} 
représentation symbolique de la nation. Il s'agit aussi de fonder la loyauté politique et l'accomplissement des devoirs civiques sur un sentiment patriotique, et pas seulement sur une adhésion de la raison. Dans cette perspective, tous les éléments d'une culture peuvent être transformés en symboles de l'identité nationale : les coutumes vestimentaires, l'histoire et la géographie, la mémoire des grands hommes, l'ensemble du patrimoine historique et culturel que l'éducation a pour tâche de transmettre aux nouvelles générations.

À vingt ans un Polonais ne doit pas être un autre homme; il doit être un Polonais. Je veux qu'en apprenant à lire il lise des choses de son pays, qu'à dix ans il en connaisse toutes les productions, à douze toutes les provinces, tous les chemins, toutes les villes, qu'à quinze il en sache toute l'histoire, à seize toutes les lois, qu'il n'y ait pas eu dans toute la Pologne une belle action ni un homme illustre dont il n'ait la mémoire et le cœur pleins, et dont il ne puisse rendre compte à l'instant ${ }^{1}$.

\section{II.2. Le pouvoir du prince et l'administration de l'État}

\section{II.2.1. Le symbole comme institution de la norme}

Si l'on se tourne maintenant vers le pouvoir du prince, et notamment vers la monarchie - à l'époque, forme prédominante de régime politique -, il faut distinguer entre pouvoir légitime et illégitime. La monarchie pour Rousseau n'est légitime que si elle respecte la souveraineté populaire et met en œuvre la volonté générale. En un mot, elle n'est légitime que dans un cadre républicain. Dans les Considérations sur le gouvernement de Pologne, on retrouve alors un usage du symbole comme opérateur d'une inversion de sens. L'intention est d'introduire une forme d'esprit républicain dans le fonctionnement de la monarchie polonaise - plus exactement, dans la réforme préconisée par Rousseau pour sauver la monarchie polonaise. On a ici un usage normatif du symbole, qui vise à instituer de nouvelles normes.

Cet usage normatif s'applique notamment au cérémonial monarchique, aux fêtes publiques et au fonctionnement de la Cour. Voici ce qu'en dit Rousseau :

$\mathrm{Ne}$ négligez pas une certaine décoration publique; qu'elle soit noble, imposante, et que la magnificence soit dans les hommes plus que dans les choses. On ne saurait croire à quel point le cœur du peuple suit ses yeux et combien la majesté du cérémonial lui en impose. Cela donne à l'autorité un air d'ordre et de règle qui inspire la confiance et qui écarte les idées de caprice et de fantaisie attachées à celle du pouvoir arbitraire ${ }^{2}$.

(418) Le cérémonial monarchique aura donc plusieurs objets : le premier est de fonder le respect du prince sur la confiance plutôt que sur la représentation de la supériorité. Le second est de faire de la régularité du protocole la représentation de la légalité. Dans les deux cas, il s'agit d'exclure de la représentation du pouvoir l'idée de l'arbitraire du prince. De ce fait, la représentation du pouvoir devient représentation de l'«autorité »: le choix de ce dernier terme résume la transformation qu'il s'agit de provoquer. Enfin, le cérémonial a aussi un troisième objet, qui est d'opérer l'inversion des valeurs dont nous avons parlé. Il s'agit de valoriser le civisme et la vertu en excluant le luxe et le clinquant des cérémonies publiques.

\footnotetext{
${ }^{1}$ Considérations sur le gouvernement de Pologne, o.c., p. 966.

${ }^{2}$ Considérations sur le gouvernement de Pologne, o.c., p. 964
} 
Il faut seulement éviter, dans l'appareil des solennités, le clinquant, le papillotage, et les décorations de luxe qui sont d'usage dans les cours. Les fêtes d'un peuple libre doivent toujours respirer la décence et la gravité, et l'on n'y doit présenter à son admiration que des objets dignes de son estime. Les Romains dans leurs triomphes étalaient un luxe énorme ; mais c'était le luxe des vaincus, plus il brillait, moins il séduisait. Son éclat même était une grande leçon pour les Romains. Les rois captifs étaient enchaînés avec des chaînes d'or et de pierreries ${ }^{1}$.

De la même façon, la cour doit donner l'exemple de la vertu patriotique. Or, l'un des pires ennemis de la vertu républicaine, c'est l'argent. Il y a une sorte d'opposition radicale entre la vertu politique et le goût des richesses. C'est pourquoi il faut bannir de la Cour l'étalage du luxe :

Tant que le luxe régnera chez les Grands, la cupidité régnera dans tous les cœurs. Toujours, l'objet de l'admiration publique sera celui des vœux des particuliers, et s'il faut être riche pour briller, la passion dominante sera toujours d'être riche ${ }^{2}$.

Ce qui vaut pour la Cour vaudra aussi pour l'administration de l'État. Là encore, les symboles utilisés à des fins politiques auront ce double aspect d'être délibérément paradoxaux et de faire prendre à l'esprit une autre direction, d'engendrer un autre « tour d'esprit ». Ainsi, Rousseau envisage pour la Pologne un système d'administration qui reposerait sur une sorte de cursus honorum. Il imagine une hiérarchie de fonctions publiques à remplir : les citoyens qui ont vocation à jouer un rôle actif dans l'État commenceraient par être avocats, assesseurs ou juges dans des tribunaux subalternes, puis députés à la Diète, puis principaux des collèges ou inspecteurs de l'éducation des enfants, enfin sénateurs. (419) Pour passer d'un grade à l'autre dans cette hiérarchie des fonctions publiques, il faudrait avoir satisfait à toutes les exigences de la fonction : probité, rigueur, sens de l'intérêt général. Les citoyens concernés porteraient sur leur vêtement des signes distinctifs indiquant leur "grade» dans cette hiérarchie politico-administrative. Or, ces signes distinctifs seront d'autant plus modestes que le grade est élevé. Les citoyens du premier échelon, ceux qui sont par exemple juges dans les tribunaux civils, porteront une plaque en or indiquant Spes patriae. Ceux qui sont députés porteront une plaque en argent portant la mention Civis electus. Ceux qui auront été sénateurs trois fois et sortiront de leur charge avec tous les honneurs entreront dans un ordre d'élite distingué par une plaque d'acier portant la mention Custos legum ${ }^{3}$. On voit que Rousseau imagine des signes distinctifs de la vertu civique. La valeur honorifique de ces signes est en raison inverse de leur valeur monétaire. Ce qu'ils signifient, c'est que la vertu civique est à l'opposé de la richesse. Pour jouir de l'honneur public, il faudra se détacher de l'or et de l'argent. Rousseau imagine ainsi un État où l'on verrait, dans la rue, passer toute sorte de citoyens portant les insignes de leurs fonctions civiques, et où ceux qui n'auraient que de l'or à montrer seraient considérés comme des subalternes.

Je voudrais que tous les grades, tous les emplois, toutes les récompenses honorifiques, se marquassent par des signes extérieurs, qu'il ne fut jamais permis à un homme en place de marcher incognito, que les marques de son rang ou de sa dignité le suivissent partout, afin que le peuple le respectât toujours, et qu'il se respectât toujours lui-même; qu'il pût ainsi toujours dominer l'opulence ; qu'un riche qui n'est que riche, sans cesse offusqué par des citoyens titrés et pauvres,

\footnotetext{
${ }^{1}$ Ibid.

${ }^{2}$ Considérations sur le gouvernement de Pologne, o.c., p. 964.

${ }^{3}$ Considérations sur le gouvernement de Pologne, o.c., pp. 1021-1023.
} 
ne trouvât ni considération ni agrément dans sa patrie ; qu'il fût forcé de la servir pour y briller, d'être intègre par ambition, et d'aspirer malgré sa richesse à des rangs où la seule approbation publique mène, et d'où le blâme peut toujours faire déchoir. Voilà comment on énerve la force des richesses, et comment on fait des hommes qui ne sont point à vendre ${ }^{1}$.

\section{II.2.2. La description critique et satirique du rituel monarchique}

Quand le monarque n'est pas seulement prince, mais veut incarner le pouvoir souverain, le régime est illégitime. La loi cesse d'être l'expression de la volonté générale pour devenir celle de la volonté du monarque. À l'usurpation du pouvoir souverain s'ajoute, dans les monarchies héréditaires, l'appropriation privée de l'État par une famille, une dynastie (420). C'est ce que montre la deuxième partie du Discours sur l'origine et les fondements de l'inégalité parmi les hommes. Un tel État est donc fondé sur la contrainte. Toutefois, l'usage de la force ne suffit pas pour garantir la soumission des sujets. Il faut qu'à la capacité de contraindre s'ajoute celle de frapper l'imagination. Il faut que le sujet, au sens politique du terme, se soumette au pouvoir en s'assujettissant à la représentation du pouvoir. Cela peut s'effectuer de plusieurs façons. Par exemple, le pouvoir peut être représenté en majesté, de telle sorte que le détenteur du pouvoir est placé au dessus du commun des mortels. La pompe et le cérémonial royaux en donnent des exemples. Le symbolisme donne aussi à imaginer un pouvoir de réserve, un pouvoir toujours en excès par rapport à l'exercice actuel de la force. Dans ce cas, c'est souvent l'appareil et l'apparat militaires qui symbolisent le pouvoir en faisant impression, en faisant la démonstration de la puissance.

Tel est le type d'analyse qu'on pourrait développer à propos du symbolisme du pouvoir en régime monarchique. Mais Rousseau ne s'attarde pas à de telles analyses. En tant qu'écrivain politique, il ne veut pas seulement démonter le fonctionnement du symbolisme monarchique. Ce qu'il veut, c'est renverser complètement la signification de ce symbolisme. À cet effet, un passage du livre II d'Émile se donne pour objet de renverser la majesté en son contraire. Le passage est tiré d'un développement où Rousseau critique les parents qui se soumettent à la tyrannie des enfants. Du thème de la tyrannie de l'enfant-roi, il passe alors à celui de l'infantilisme du tyran. Suit l'exemple de la monarchie héréditaire où l'héritier du trône est souvent un enfant en bas âge. Voici l'extrait :

Je comprends que le rugissement d'un lion épouvante les animaux, et qu'ils tremblent en voyant sa terrible hure; mais si jamais on vit un spectacle indécent, odieux, risible, c'est un corps de magistrats le chef à la tête en habit de cérémonie, prosternés devant un enfant au maillot qu'ils haranguent en termes pompeux et qui crie et bave pour toute réponse ${ }^{2}$.

On voit comment Rousseau retourne ici la signification du cérémonial monarchique. Ce cérémonial est ridicule parce qu'il est contre nature. Il est contre nature que les adultes obéissent à un enfant. Alors que le symbolisme politique est censé mettre en scène la majesté, la description de ce symbolisme fonctionne ici à contresens. Le rituel de la majesté est infantile. Ce renversement culmine dans la description de "l'échange verbal». Il y a une parole dominante et une parole dominée. Mais la parole dominée, c'est la harangue des grands du royaume. Quant à la parole dominante, c'est celle d'un roi en couches-culottes. Ce n'est

\footnotetext{
${ }^{1}$ Considérations sur le gouvernement de Pologne, o.c., p. 1007.

${ }^{2}$ Émile, Livre IV, o.c., p. 315.
} 
d'ailleurs pas une véritable parole. C'est une éructation qui, au lieu d'articuler du sens, ne produit que de la bave et des cris.

(421) Nous avons ici une description renversante du symbolisme du pouvoir, un usage à contresens du symbolisme. Cet usage illustre le double fonctionnement du symbole comme acte que nous avons analysé plus haut. D'une part, Rousseau condense dans une image très contrastée, en quelques lignes, une série de significations qui agissent directement sur l'esprit du lecteur. D'autre part, cette image crée chez le lecteur un état d'esprit adapté à la réception des arguments critiques développés par ailleurs contre la monarchie héréditaire, système où le peuple risque d'avoir «pour chefs des enfants, des monstres, des imbéciles ${ }^{1}$. Le sentiment du profond ridicule de la pompe monarchique correspond à un état d'esprit républicain.

\section{Conclusion}

Voilà quelques usages du symbolisme politique chez Rousseau. Le point commun à ces usages est la vocation éducative de la représentation du pouvoir. Il y a chez Rousseau une sorte de pédagogie politique qui doit porter sur le désir, c'est-à-dire, sur les représentations collectives qui orientent les désirs des citoyens. "Quiconque se mêle d'instituer un peuple doit savoir dominer les opinions et par elles gouverner les passions des hommes $»^{2}$. Cette pédagogie politique et, d'une manière générale, l'usage des représentations symboliques sont liés à une conscience très vive des limites de l'argumentation. Ces limites apparaissent de différentes manières. D'une part, l'adhésion aux principes constitutifs de l'État ne peut pas être une adhésion purement rationnelle, elle suppose une certaine forme de sensibilité. D'autre part, ce que l'argumentation gagne en rigueur logique, elle le perd en force d'expression. Il faut donc agir sur les deux plans. C'est pourquoi Rousseau a élaboré un style argumentatif où l'image, le paradoxe, les formules contrastées, agissent sur l'esprit du lecteur à la façon de gestes. Enfin, il y a dans l'usage rousseauiste du symbole politique une réflexion sur la réception. Un argument ne porte que si le destinataire le reçoit de la manière appropriée. S'il ne crée pas les conditions de sa propre réception, l'argument est sans effet. Ce principe vaut également pour l'argumentation politique et pour toute forme de gouvernance reposant sur l'usage de la parole. Dès lors, il s'agit de substituer, au symbole mystificateur par lequel s'impose le pouvoir arbitraire, un symbolisme éducateur qui fonctionne à contre-sens : un symbolisme qui, en réorientant le désir autant que la pensée, pourrait contribuer à réformer l'État ou, tout au moins, à ralentir son inévitable déclin.

\footnotetext{
${ }^{1}$ Du contrat social, Livre III, chapitre VI, « De la monarchie », o.c., p. 411.

${ }^{2}$ Considérations sur le gouvernement de Pologne, o.c., p. 965-966.
} 